\title{
Network oscillations at the boundary of an equatorial coronal hole
}

\author{
H. Tian ${ }^{1,2}$ and L.-D. Xia ${ }^{3}$ \\ 1 Max-Planck-Institut für Sonnensystemforschung, Katlenburg-Lindau, Germany \\ e-mail: tian@mps.mpg.de \\ 2 Department of Geophysics, Peking University, Beijing, PR China \\ 3 School of Space Science and Physics, Shandong Univ. at Weihai, Weihai, Shandong, PR China \\ e-mail: xld@sdu.edu.cn
}

Received 5 May 2008 / Accepted 20 June 2008

\section{ABSTRACT}

\begin{abstract}
Aims. We investigate intensity oscillations observed simultaneously in the quiet chromosphere and in the corona, above an enhanced network area at the boundary of an equatorial coronal hole.

Methods. A Fourier analysis is applied to a sequence of images observed in the $171 \AA$ and $1600 \AA$ passbands of TRACE. Four interesting features above the magnetic network are further investigated by using a wavelet analysis.

Results. Our results reveal that, in both the $171 \AA$ and $1600 \AA$ passbands, oscillations above the magnetic network show a lack of power at high frequencies $(5.0-8.3 \mathrm{mHz})$, and a significant power at low $(1.3-2.0 \mathrm{mHz})$ and intermediate frequencies $(2.6-4.0 \mathrm{mHz})$. The global 5-min oscillation is clearly present in the 4 analyzed features when seen in the $1600 \AA$ passband, and is also found with enhanced power in feature 1 (leg of a large coronal loop) and feature 2 (legs of a coronal bright point loop) when seen in the $171 \AA$ passband. Two features above an enhanced network element (feature 3 and feature 4) show repeated propagating behaviors with a dominant period of $10 \mathrm{~min}$ and $5 \mathrm{~min}$, respectively.

Conclusions. We suggest these oscillations are likely to be slow magneto-acoustic waves propagating along inclined magnetic field lines, from the lower solar atmosphere into the corona. The energy flux carried by these waves is estimated of the order of $40 \mathrm{erg} \mathrm{cm}^{-2} \mathrm{~s}^{-1}$ for the $171 \AA$ passband and is far lower than the energy required to heat the quiet corona. For the $1600 \AA$ passband, the energy flux is about $1.4 \times 10^{6} \mathrm{erg} \mathrm{cm}^{-2} \mathrm{~s}^{-1}$, which is about one third of the required energy budget for the chromosphere.
\end{abstract}

Key words. Sun: oscillations - Sun: corona - Sun: chromosphere - Sun: UV radiation

\section{Introduction}

The solar atmosphere is highly structured by the magnetic field. The chromospheric network, which is the upward extension of the supergranular boundaries above the photosphere, is characterized by clusters of magnetic flux concentrations (Gabriel 1976). Part of the network flux expands with height (Patsourakos et al. 1999; Tian et al. 2008a) and opens into the corona with a funnel shape, while the rest of the network consists of a dense population of low-lying loops with lengths less than $10^{4} \mathrm{~km}$ (Dowdy et al. 1986; Peter 2001).

The observation of oscillations in the chromosphere and corona can help us understand the magnetic structure of the solar atmosphere, and provides valuable insight into the unresolved coronal heating problem.

It has been shown that oscillations with different frequencies $(0.6 \mathrm{mHz}-2.7 \mathrm{mHz})$ are present in the chromospheric network (Curdt \& Heinzel 1998; Lites et al. 1993; Curdt \& Heinzel 1998; Cauzzi et al. 2000). McAteer et al. (2002) found waves with a period of $4-15 \mathrm{~min}(1-4 \mathrm{mHz})$ in the central portions of network bright points, and suggest that these waves are possibly magneto-acoustic or magneto-gravity modes. More recently, McAteer et al. (2004) found that the most frequent network oscillation has a period of $283 \mathrm{~s}$, with a lifetime of 2-3 cycles in four TRACE (Transition Region and Coronal Explorer) passbands centered at $1700,1600,1216$, and $1550 \AA$. The power of the intensity fluctuations in the $1600 \AA$ passband (UV continuum at $4-10 \times 10^{3} \mathrm{~K}$ plus Fe II at $1.3 \times 10^{4} \mathrm{~K}$ ), which is formed at the temperature minimum (McAteer et al. 2004), is found to peak at $3-5 \mathrm{mHz}$ and considered as a mixture of photospheric 5-min oscillations and chromospheric 3-min oscillations (Fossum \& Carlsson 2005). The so-called "magnetic shadows", which surround magnetic network elements and show a lack of oscillatory power in the period range of 2-3 min, have received much attention (McIntosh \& Judge 2001; Krijger et al. 2001; Vecchio et al. 2007) and have been considered to play an important role in the trapping of high-frequency magneto-acoustic oscillations (Srivastava et al. 2008).

Oscillations with different periods have also been found in different regions above the chromosphere. In the active region, 3-min oscillations have been found in the transition region and corona in sunspot regions (Fludra 1999; Brynildsen et al. 1999; De Moortel 2002), while 5-min oscillations are more likely to be found in "non-sunspot" loops (De Moortel 2002) and above AR plage (De Pontieu 2003, 2005). These oscillations are suggested to be due to upward-propagating slow magneto-acoustic waves. The 5-min oscillation is likely to be guided along inclined magnetic flux tubes, which can decrease the acoustic cutoff frequency to allow the low-frequency photospheric oscillations propagate into the outer atmosphere (De Pontieu 2004; McIntosh \& Jefferies 2006; Hansteen et al. 2006). In the open-field regions, quasi-periodic perturbations with periods of 10-15 min (DeForest \& Gurman 1998) and 10-25 min (Banerjee et al. 2000) have been found in polar plumes. These low-frequency perturbations are explained as compressive waves (such as sound waves or slow-mode magneto-acoustic waves) 
propagating along the plumes (Ofman et al. 1999). In spicules, the 5-min oscillation is also present (Xia et al. 2005), and might be driven by p-modes (De Pontieu 2004). By measuring phase delays between intensity oscillations and between velocity oscillations of different line pairs, O'Shea et al. (2007) concluded that propagating slow magneto-acoustic waves are present in coronal holes and that they occur preferentially in bright regions that are associated with magnetic field concentrations in the form of loops or bright points. Periodic oscillations (7-64 min) in coronal bright points have also been found by Ugarte-Urra et al. (2004a), Ugarte-Urra et al. (2004b), and Tian et al. (2008b). By using the new XRT data, Kariyappa \& Varghese (2008) recently found that X-Ray bright point emission shows several significant peaks at different frequencies corresponding to time scales that range from a few minutes to hours.

In this contribution, we investigate intensity oscillations observed simultaneously in the quiet chromosphere and in the corona, above an enhanced network area at the boundary of an equatorial coronal hole.

\section{Observations and data analysis}

The data set analyzed here contains a sequence of images observed from 07:40 to 08:20 UTC on November 08 in 1999 in two passbands (1600 $\AA$ and $171 \AA$ ) of the TRACE instrument. The emissions of the $1600 \AA$ and $171 \AA$ passbands are formed in the chromosphere (at temperature minimum) and in the lower corona, respectively. The sequence of $171 \AA$ only lasted from 07:40 to 08:12. The pixel size is $0.5^{\prime \prime}$ for the $1600 \AA$ images, and $1.0^{\prime \prime}$ for the $171 \AA$ images. Standard software for calibrating and correcting the TRACE data was applied to this data set, including removal of cosmic rays, subtraction of the dark current, normalization of the counts, and so on. We used the cross correlation technique to do the coalignment of the images in the sequence.

We extracted a sequence of sub-images with a size of $130^{\prime \prime} \times$ $95^{\prime \prime}$ from the original data set. The sub-images were taken around the boundary of an equatorial coronal hole above an enhanced network area. The average intensity images of the studied region in the $1600 \AA$ and $171 \AA$ passbands can be found in Figs. $2 \mathrm{~A}$ and $\mathrm{E}$, respectively. The chromospheric network pattern is clearly seen in the $1600 \AA$ passband. The coronal hole and the surrounding quiet Sun can be easily discerned in the image of the $171 \AA$ passband. Figure 1 shows the magnetogram corresponding to the studied region.

The cadence of our TRACE data was about $30 \mathrm{~s}$. At a few moments there were irregular gaps. This problem was overcome by applying a linear interpolation with the help of the INTERPOL function in the IDL software. We then created a sequence of running difference images, by subtracting each image from the image taken $30 \mathrm{~s}$ earlier. This sequence of running difference images was further analyzed to search for periodic signatures.

We applied a standard Fourier analysis to the sequence of running difference images of each passband. At each spatial pixel, we obtained a frequency-power curve. Then we added the total power respectively in three different frequency ranges, and calculated the percentage of this value relative to the total power in the full frequency range. In this way we obtained a relative power map in each frequency range. The three frequency ranges are $1.3-2.0 \mathrm{mHz}(500-780 \mathrm{~s}$, low frequencies), $2.6-4.0 \mathrm{mHz}$ (250-384 s, intermediate frequencies), and

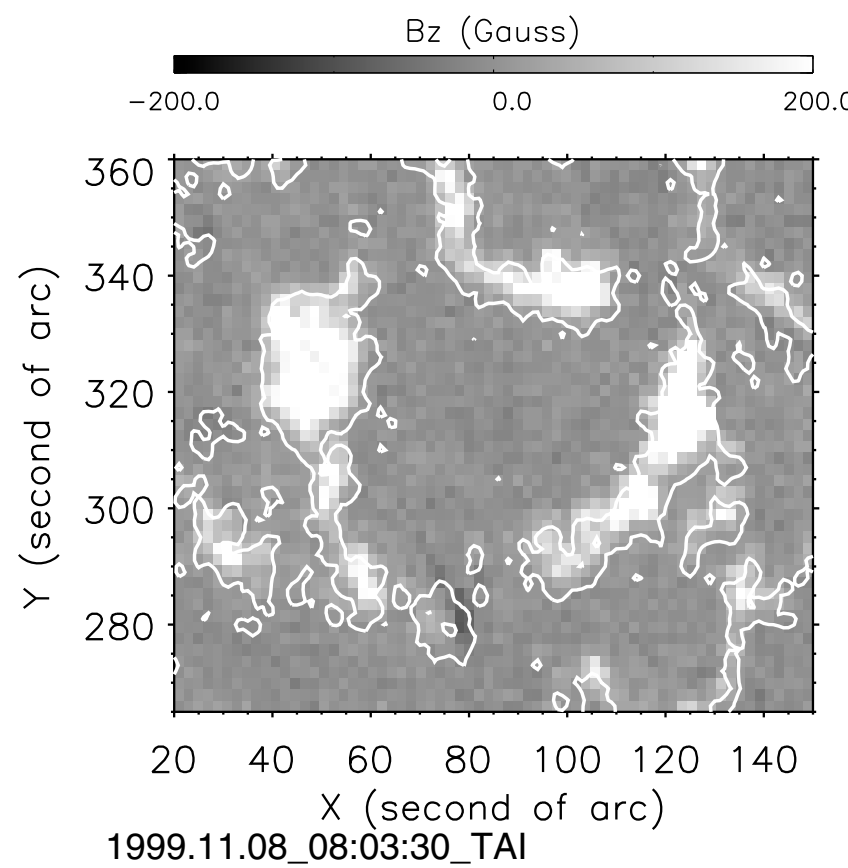

Fig. 1. MDI magnetogram of the studied region taken at 08:03:30 UTC on November 08 in 1999. The white contours correspond to bright lanes seen in the averaged image of the $1600 \AA$ passband.

5.0-8.3 mHz (120-200 s, high frequencies). The power maps can be found in Fig. 2.

The intensity images and maps of Fourier power reveal several interesting features. We chose four features for a more detailed analysis. They are shown in Fig. 2 and outlined in white. By checking the original TRACE images and the full-disk MDI magnetogram, we found that feature 1 might be a leg of a large coronal loop, feature 2 is a coronal bright point. Feature 3 and feature 4 are above an enhanced network element and their enhanced power forms an elongated shape at low and intermediate frequencies, respectively.

We averaged the intensity in the outlined rectangular area for each feature, and created the corresponding running difference light curve. These curves are shown in the upper panels of Fig. 4 to Fig. 7. Then we applied a Fourier analysis and a wavelet analysis to each running difference light curve. The Fourier spectra are shown in Fig. 3. The solid and dashed curves correspond to the power of the $1600 \AA$ and $171 \AA$ passband, respectively.

The localized nature of the wavelet transform allows us to study the duration of any statistically significant oscillations as well as their period (Banerjee et al. 2000). By decomposing a time series into time-frequency space, one is able to determine both the dominant modes of variability and how those modes vary in time (Torrence \& Compo 1998). Here we also performed a wavelet analysis for the running difference light curves of the four features, in order to find the most reliable periods. We chose the Morlet wavelet function, defined as a sine wave modulated by a Gaussian window, for our analysis. The wavelet transform suffers from edge effect at both ends of the time series. And this effect is important in regions defined as the "cone of influence" (COI). To check whether the periodic signatures present in the wavelet spectrum are real or not, we have to perform a significance test. Here we chose a confidence level of $95 \%$. The description of the wavelet function, COI, and significance test can be found in Torrence \& Compo (1998). 

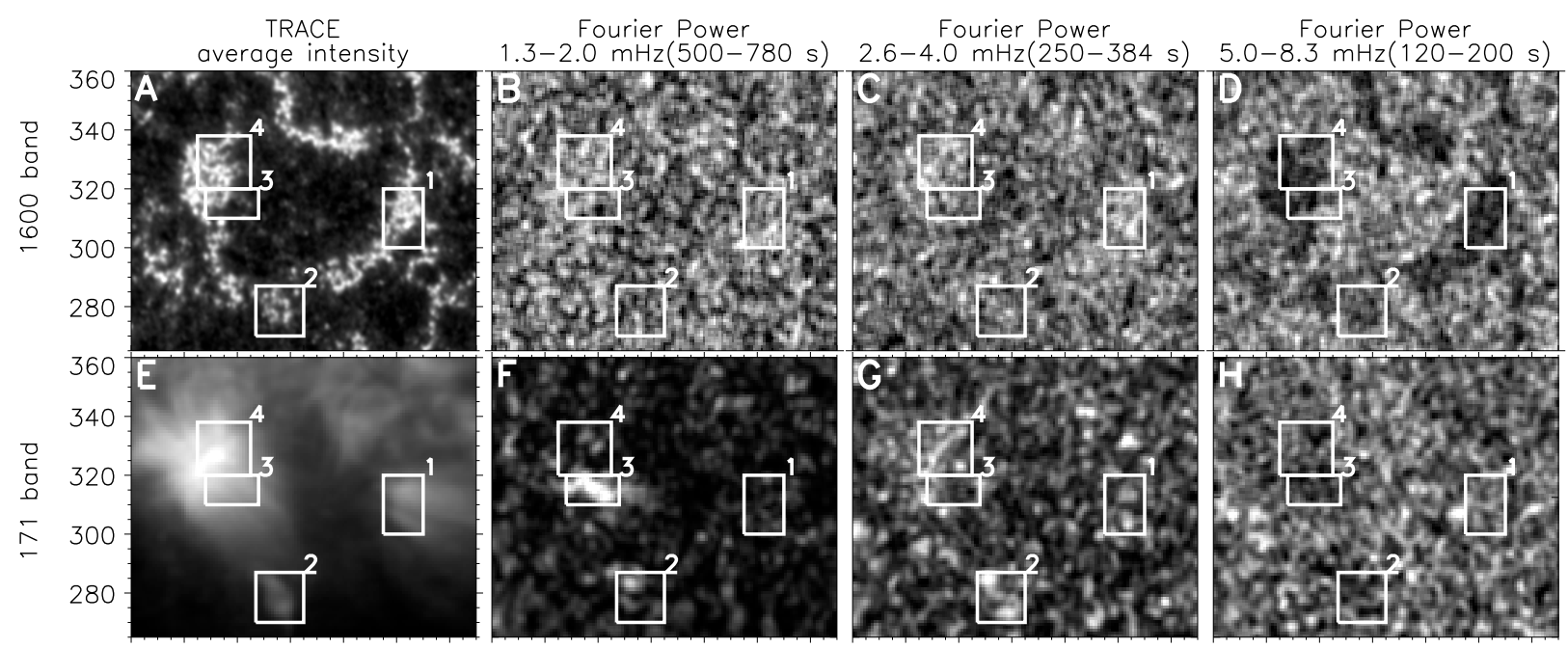

$2040 \quad 60801001201402040 \quad 60 \quad 8010012014020 \quad 40 \quad 608010012014020 \quad 40 \quad 60 \quad 80100120140$

Fig. 2. Panel A-D: the average intensity image of the $1600 \AA$ passband, and maps of Fourier power in frequency ranges of $1.3-2.0 \mathrm{mHz}$, 2.6-4.0 mHz, and 5.0-8.3 mHz. Panel E-H: similar to panel A-D, but for the 171 A passband.
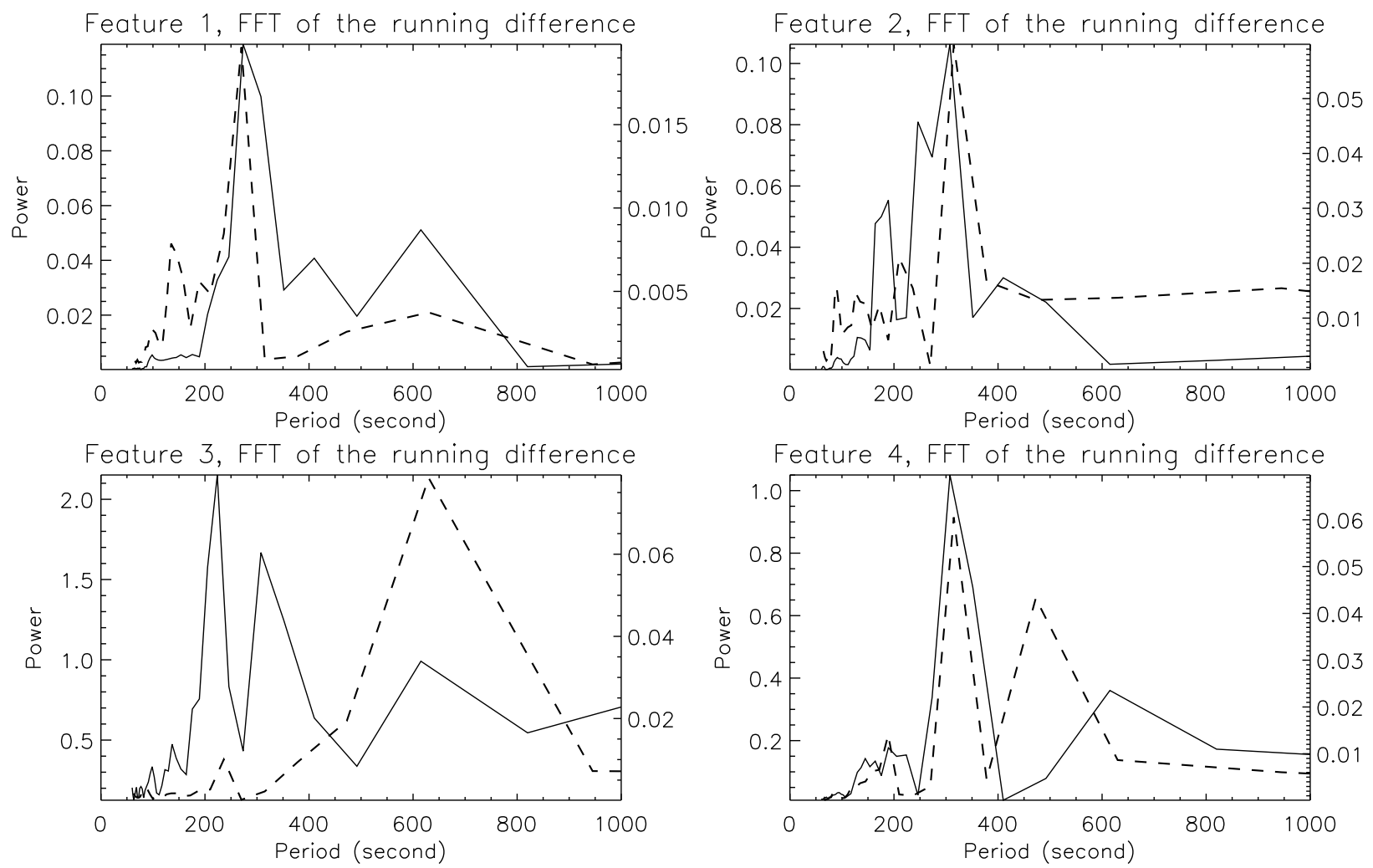

Fig. 3. The Fourier spectra for the four features. The solid and dashed curves correspond to the power of the $1600 \AA$ and $171 \AA$ passbands and their scales are given on the left-hand and right-hand $y$-axes, respectively.

The wavelet power spectra of the four features are shown in Fig. 4 to Fig. 7. Cross-hatched regions indicate the "cone of influence". The darker parts represent higher power, and the contours correspond to the $95 \%$ confidence level. We also averaged each wavelet power spectrum over time and plotted the global wavelet spectrum.

\section{Results and discussion}

The main motivation of this work is to search for the signature of oscillations at the boundary of an equatorial coronal hole, and to study oscillations observed almost simultaneously in the chromosphere and corona above the magnetic network. The Fourier and wavelet analyses show that obvious oscillations really exist in our data.

Figures. 2B-D show the maps of Fourier power in different frequency ranges in the $1600 \AA$ passband. It is very clear that the network oscillations reveal a lack of power at high frequencies, and a significant power at low and intermediate frequencies. The reason why the power at high frequencies in the internetwork region is stronger than that in the network lane might 


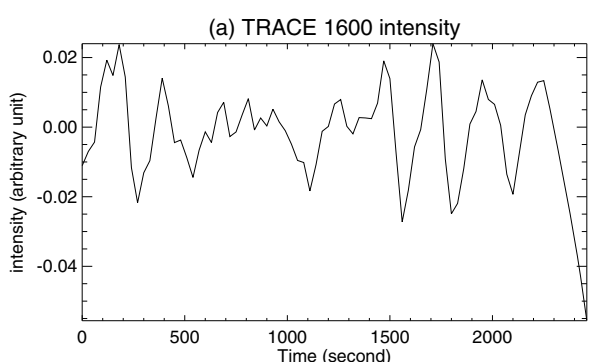

(b) Wavelet Power Spectrum

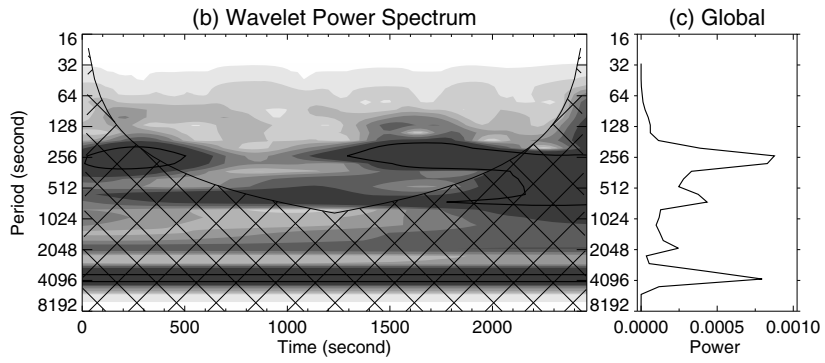

(a) TRACE 171 intensity

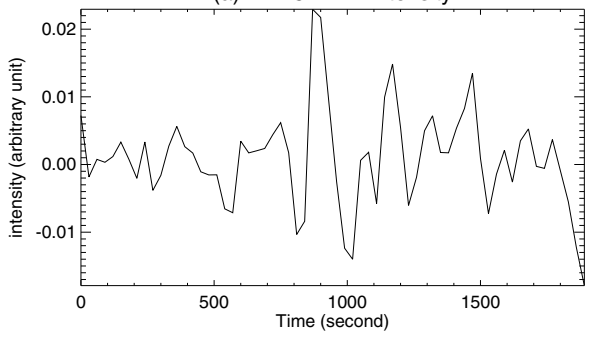

(b) Wavelet Power Spectrum

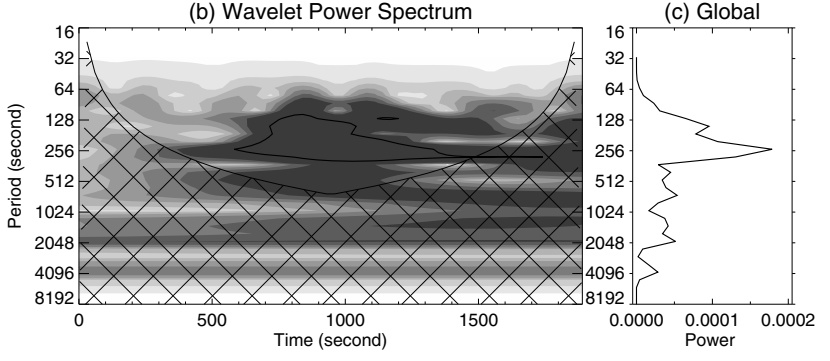

Fig. 4. Wavelet power spectra of the $1600 \AA$ (left $)$ and $171 \AA$ (right) passbands for feature 1. (a) The running difference light curve. (b) Time/period variation of the wavelet power spectrum. Cross-hatched regions indicate the "cone of influence". The darker parts represent higher power, and the contours correspond to the $95 \%$ confidence level. (c) Global wavelet.

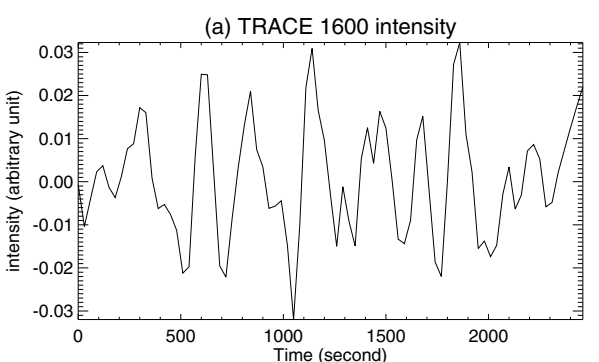

(b) Wavelet Power Spectrum

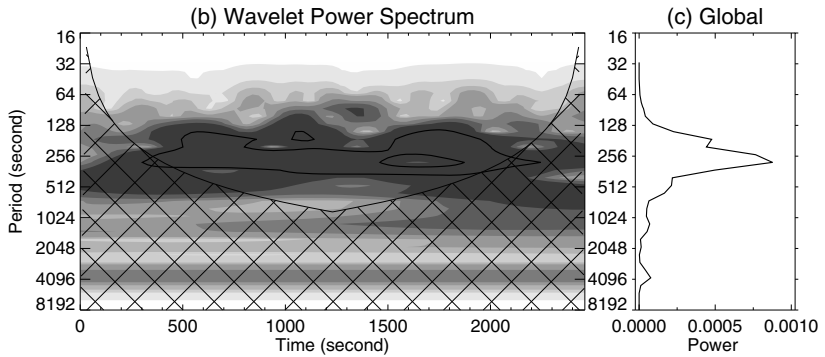

(a) TRACE 171 intensity

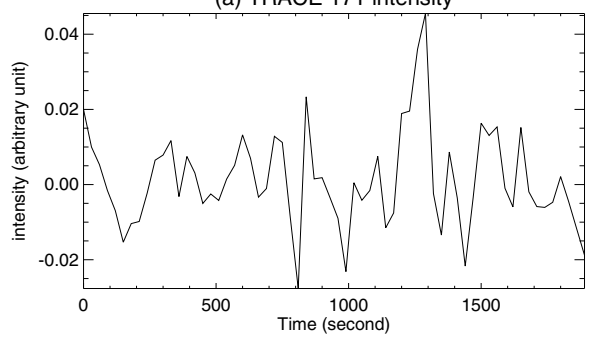

(b) Wavelet Power Spectrum

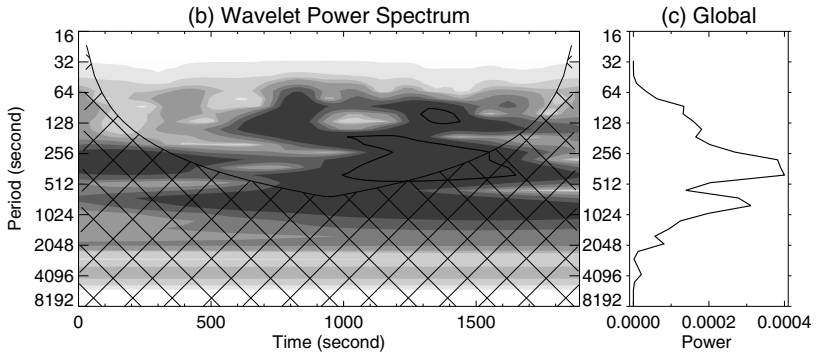

Fig. 5. Same as Fig. 4 but for feature 2.

be a result of the network magnetic structure. It is known that part of the network flux expands with height and opens into the corona (Dowdy et al. 1986; Peter 2001; Patsourakos et al. 1999; Tian et al. 2008a). Thus, photospheric oscillations with frequencies above the acoustic cutoff (about $5.5 \mathrm{mHz}$ ) can propagate into the chromosphere, where the network fluxes begin to stretch into the region above the internetwork in the shape of canopies (Srivastava et al. 2008). Below the canopy field, magnetic shadows with reduced oscillatory power at high frequencies are present within and immediately around the magnetic network (Vecchio et al. 2007). But the significant power (seen in Ca II $854.2 \mathrm{~nm}$ ) directly above the magnetic network in Vecchio et al. (2007) is not very clear in our data set, which might be due to the different formation height between Ca II and the $1600 \AA$ passband of TRACE, or due to the relatively low spatial resolution of our data set.

The result of a significant power at low and intermediate frequencies might be explained as a leakage of low-frequency photospheric oscillations into the chromosphere through "magneto-acoustic portals" which are positioned within the network. It might provide a significant energy source to heat the quiet chromosphere (Jefferies et al. 2006; Vecchio et al. 2007).

As for the power maps of the $171 \AA$ passband, we can make a similar conclusion to that of the $1600 \AA$ passband. But the significant powers at low and intermediate frequencies are more constricted and isolated in the network lane. Also, the regions with reduced power at high frequencies are not so prominent and are more diffused. It might be a natural result of the diffusion and expansion of the magnetic structure from the chromosphere to the corona.

As we stated above, feature 1 corresponds to a leg of a large coronal loop system. Figures $2 \mathrm{C}$ and $2 \mathrm{G}$ reveal a significant power at intermediate frequencies in this loop leg, both in the $1600 \AA$ and $171 \AA$ passbands. The period corresponding to these frequencies is around $5 \mathrm{~min}$. Although oscillations with periods 180-420 s have already been reported by De Moortel (2000), it 


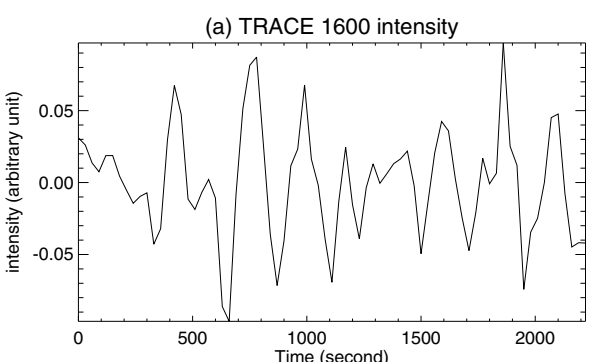

(b) Wavelet Power Spectrum

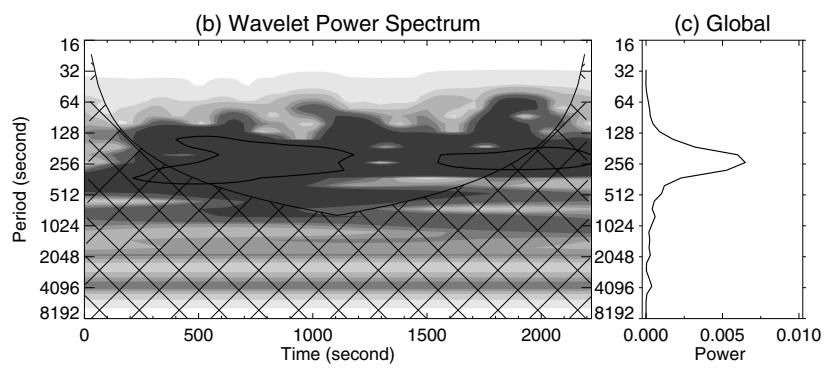

Fig. 6. Same as Fig. 4 but for feature 3.
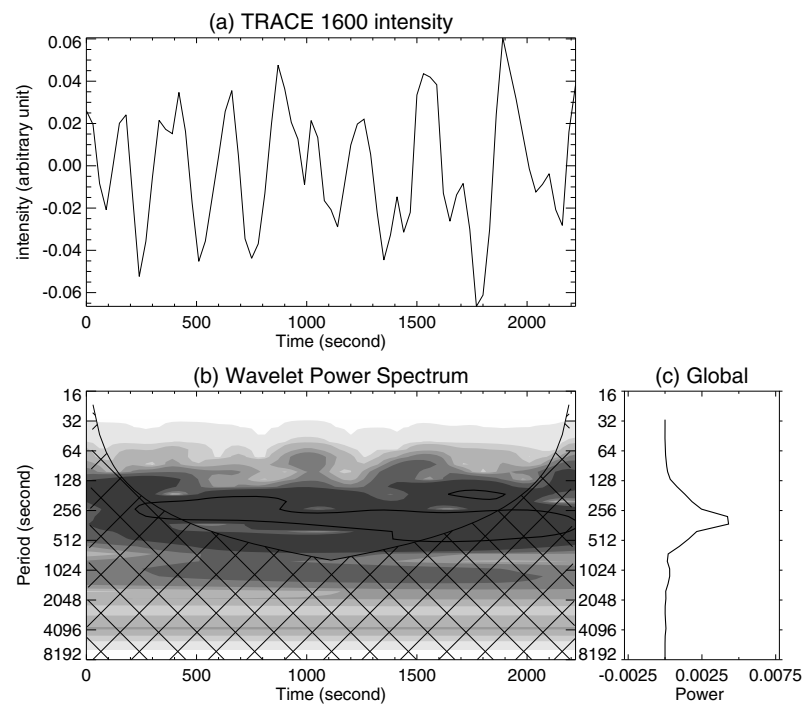

Fig. 7. Same as Fig. 4 but for feature 4.

is still very interesting to note that in feature 1 the two bright dots seen in Fig. 2G correspond exactly to the two parts of the loop leg seen in Fig. 2E. This result indicates that in a loop system, the strongest power of the 5-min oscillation locates at the lower part of the leg. As discussed in De Moortel (2000), the 5-min oscillation might be due to the propagating slow magneto-acoustic waves. From Figs. 3 and 4, we find that the power peak around 5 min is clearly present at most of the time. There is a weak peak at 10 min for the Fourier spectra in both the $1600 \AA$ and $171 \AA$ passbands, but not significant in the wavelet power spectra.

Feature 2 corresponds to a coronal bright point, which is characterized by an enhanced coronal emission associated with bipolar magnetic field. Ugarte-Urra et al. (2004a) and UgarteUrra et al. (2004b) studied periodic oscillations of coronal bright points, and found oscillations with a period of 400-1100 s. Longer period (8-64 min) oscillations were also detected by Tian et al. (2008b). Here from Figs. 3 and 5 we find that the oscillatory power of this bright point has a major peak around $5 \mathrm{~min}$, and a second peak around $3 \mathrm{~min}$. If we compare Figs. $2 \mathrm{E}$ and $2 \mathrm{G}$, we can find that the strongest power of feature 2

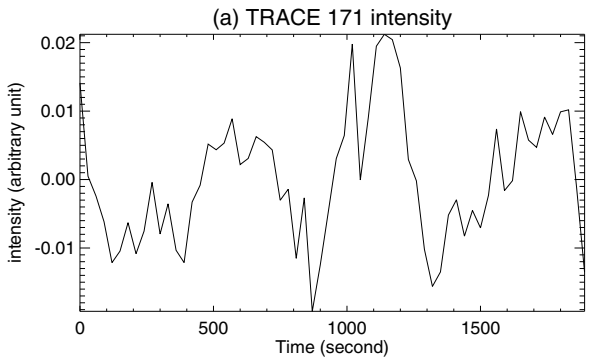

(b) Wavelet Power Spectrum

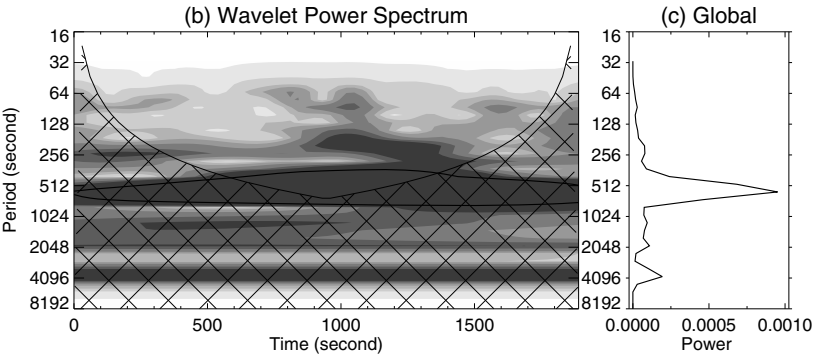

(c) Global
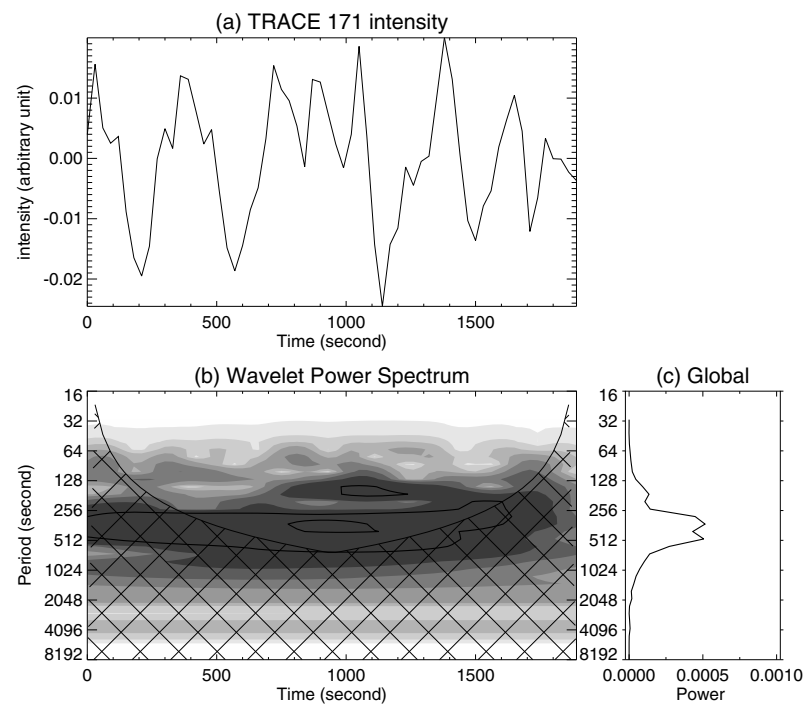

corresponds to the two parts of the bright emission. These two parts might be the two legs of the loop system associated with the bright point.

Feature 3 locates above the outer part of an enhanced network element. The most interesting point is that the enhanced Fourier power at low frequencies has an obvious elongated shape in the $171 \AA$ passband. The movie of the image sequence clearly reveals that a periodic motion is present in this region, and the direction of this motion is exactly along this elongated shape. The Fourier and wavelet analyses both reveal a 10-min oscillation in the $171 \AA$ passband. This oscillation is present in the entire duration of the time series, which can be seen in Fig. 6. While in the $1600 \AA$ passband, the Fourier spectrum shows three peaks at 3 , 5 , and $10 \mathrm{~min}$. However, only the 3-min and 5-min oscillations are considered as significant in the wavelet power spectrum. It has been known that strongly inclined magnetic fields can significantly decrease the acoustic cut-off frequency (De Pontieu 2004; McIntosh \& Jefferies 2006; Hansteen et al. 2006), and thus allow high-frequency photospheric oscillations (above the acoustic cut-off frequency $5.5 \mathrm{mHz}$ ) to propagate into the 
chromosphere and corona. Figure $2 \mathrm{E}$ gives us the impression that the magnetic field of feature 3 seems to be strongly inclined, and thus is very likely to guide the high-frequency oscillations from the lower part of the solar atmosphere to the corona. The weak peak at $10 \mathrm{~min}$ in the Fourier spectrum of the $1600 \AA$ passband, although not prominent in the wavelet spectrum, might still be the counterpart of the dominant 10-min oscillation seen in the $171 \AA$ passband.

As for feature 4, which is above the same enhanced network element as feature 3 but in a different location, the power peak around $5 \mathrm{~min}$ is also clear in both the Fourier and the wavelet spectra for each bandpass. From Fig. 7 we can see that in the $1600 \AA$ passband the period is initially around $4 \mathrm{~min}$, then increases to about $6 \mathrm{~min}$ in the second half of the duration. In the $171 \AA$ passband, a period of 5-8 min is present through almost the entire duration. The strong power of the 5-min oscillation also seems to form an elongated shape in the $171 \AA$ passband shown in Fig. 2G. A periodic motion is also present along this elongated direction when seen in the movie of the image sequence.

The propagation speeds can be estimated if we plot the running difference along the long side of the bars shown in the upper panels of Fig. 8, which indicate the directions of the propagating 10-min oscillation in feature 3 and 5-min oscillation in feature 4 in the $171 \AA$ passband. These plots are demonstrated in the low panels of Fig. 8. The slope of the dashed line provides an estimate of the propagating speed, which is about $32 \mathrm{~km} \mathrm{~s}^{-1}$ for the 10-min oscillation, and $58 \mathrm{~km} \mathrm{~s}^{-1}$ for the 5-min oscillation. These values are lower than the propagating speed of the slow magneto-acoustic waves derived by De Moortel (2000) and DeForest \& Gurman (1998), which are approximately $70-165 \mathrm{~km} \mathrm{~s}^{-1}$ at a bright loop-footpoint and 75$150 \mathrm{~km} \mathrm{~s}^{-1}$ in polar plumes, respectively. However, taking into account the line-of-sight effect of the measured propagating speed, our values are still of the order of the coronal sound speed, which is about $150 \mathrm{~km} \mathrm{~s}^{-1}$ (De Moortel 2000). Since Alfvén oscillations are essentially velocity perturbations and do not reveal themselves in intensity fluctuations, our periodic propagating signatures are most likely to be slow magneto-acoustic waves.

Here we will roughly estimate the energy flux carried by these waves. We simply take the relationship in Sakurai et al. (2002):

$\frac{\delta I}{I}=2 \frac{\rho^{\prime}}{\rho}=2 \frac{\delta v}{C_{\mathrm{s}}}$,

where $\delta I / I, \rho^{\prime} / \rho, \delta v$ and $C_{\mathrm{s}}$ are intensity fluctuation, density fluctuation, wave velocity amplitude, and sound speed, respectively. In our data, we found a value of about 0.015 for $\delta I / I$. Given the sound speed of $150 \mathrm{~km} \mathrm{~s}^{-1}$ at the formation temperature of the $171 \AA$ passband, we can calculate the wave velocity amplitude as $1.05 \mathrm{~km} \mathrm{~s}^{-1}$. Following Ofman et al. (1999), the energy flux carried by slow magneto-acoustic waves can be calculated in the following way:

$F=0.5 \rho(\delta v)^{2} C_{\mathrm{s}}$,

where $F$ and $\rho$ represent energy flux and density, respectively. By using $\rho=5 \times 10^{-16} \mathrm{~g} \mathrm{~cm}^{-3}$ (De Moortel 2000) and substituting the values of $\rho$ and $\delta v$, we obtained an energy flux of about $40 \mathrm{erg} \mathrm{cm} \mathrm{cm}^{-2} \mathrm{~s}^{-1}$ for the propagating oscillations. These values are much lower than the required heat input to the quiet corona, which is about $3 \times 10^{5} \mathrm{erg} \mathrm{cm}^{-2} \mathrm{~s}^{-1}$ in Withbroe \& Noyes (1977). Thus, these waves are not appropriate candidates for coronal heating.
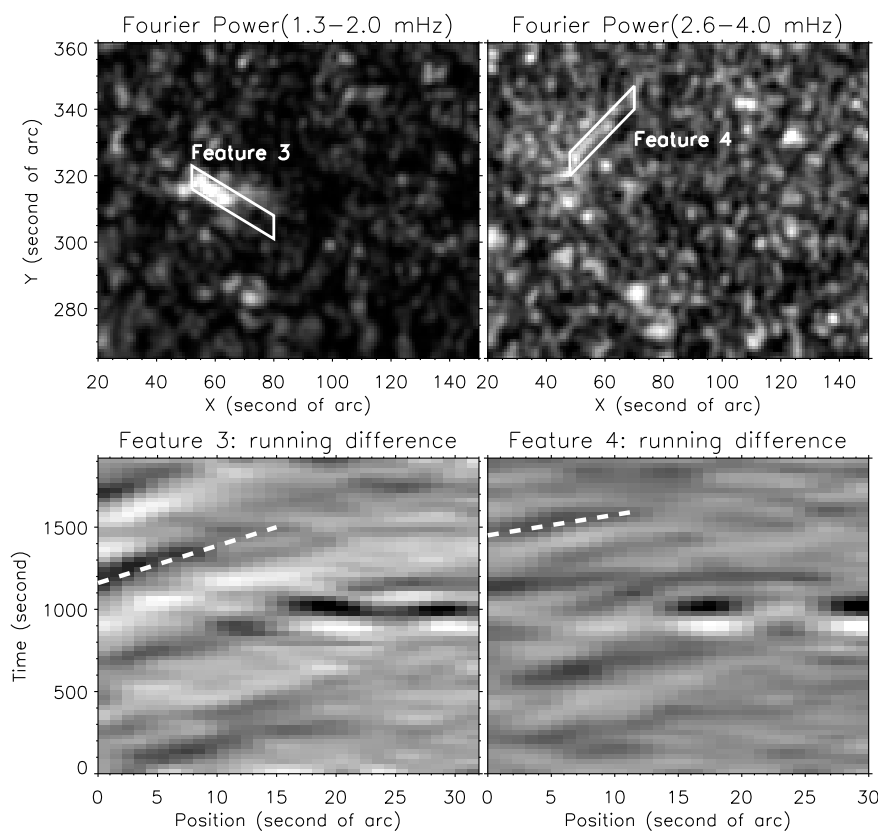

Fig. 8. Upper: the same as Fig. 2F and Fig. 2G. The bars indicate the propagating directions of the oscillations. Lower: plots of running difference along the long sides of the bars shown in the upper panels. The dashed lines are used to calculate the speed of propagating oscillations.

For the chromospheric oscillation, if we take the value of 0.04 for $\delta I / I$ and the sound speed of $16 \mathrm{~km} \mathrm{~s}^{-1}$ at the formation temperature of the $1600 \AA$ passband, we can calculate the wave velocity amplitude as $0.32 \mathrm{~km} \mathrm{~s}^{-1}$ by using Eq. (1). Given a particle number density of $10^{21} \mathrm{~cm}^{-3}$ at the temperature minimum (Peter 2004), we can calculate the proton mass density $\rho$. By substituting the values of $\rho, \delta v$, and $C_{\mathrm{s}}$ in the chromosphere to Eq. (2), we obtain a value of $1.368 \times 10^{6} \mathrm{erg} \mathrm{cm}^{-2} \mathrm{~s}^{-1}$ for the energy flux carried by the waves at the emission height of the $1600 \AA$ passband. This value is very close to that estimated by Jefferies et al. (2006) and is almost one third of the required energy budget for the chromosphere. Thus, our results support the conclusion in Jefferies et al. (2006) that low-frequency magnetoacoustic waves provide a significant source of energy for balancing the radiative losses of the ambient solar chromosphere.

Finally, we have to point out that the relatively short length (low amount) of data restricts our discussion and lowers the solidness of the results. In order to fully understand network oscillations at boundaries of equatorial coronal holes, deep investigation to more data sets is required in the future.

\section{Summary and conclusion}

With the help of Fourier and wavelet analyses, we studied intensity oscillations observed simultaneously in the quiet chromosphere and corona, above an enhanced network area at the boundary of an equatorial coronal hole.

Images of Fourier power reveal that, oscillations above the magnetic network show a lack of power at high frequencies $(5.0-8.3 \mathrm{mHz})$, and a significant power at low $(1.3-2.0 \mathrm{mHz})$ and intermediate frequencies $(2.6-4.0 \mathrm{mHz})$ in both the $171 \AA$ and $1600 \AA$ passbands. The former result suggests that "magnetic shadows" not only exist in the chromosphere, but also extend into the lower corona. The latter result supports the concept of "magneto-acoustic portals" within the network through which 
low-frequency photospheric oscillations can propagate into the chromosphere and corona.

We also studied 4 interesting features in more detail. The global 5-min oscillation is clearly present in all of the 4 analyzed features when seen in the $1600 \AA$ passband, and is also found with enhanced power in feature 1 (leg of a large coronal loop) and feature 2 (legs of a coronal bright point loop) when seen in the $171 \AA$ passband. Two features above an enhanced network element (feature 3 and feature 4) show repeated propagating behaviors with a dominant period of $10 \mathrm{~min}$ and $5 \mathrm{~min}$, respectively. The derived values of the propagating speed are of the order of the coronal sound speed. These velocities, together with the compressional nature of the oscillation, suggest that our periodic propagating signatures are most likely to be slow magneto-acoustic waves.

We calculated the energy flux carried by these waves and found a value of about $40 \mathrm{erg} \mathrm{cm}^{-2} \mathrm{~s}^{-1}$ for the $171 \AA$ passband, which is only a small fraction of the total energy required to heat the quiet corona. However, for the $1600 \AA$ passband, the energy flux is about $1.368 \times 10^{6} \mathrm{erg} \mathrm{cm}^{-2} \mathrm{~s}^{-1}$, which is of the order of the required energy budget for the chromosphere.

Acknowledgements. The TRACE satellite is a NASA Small Explorer that images the solar photosphere, transition region and corona with unprecedented spatial resolution and temporal continuity. We thank $\mathrm{C}$. Torrence and $\mathrm{G}$. P. Compo for providing the Wavelet software, which is available at URL: http://paos.colorado.edu/research/wavelets/. We also thank Dr. Zhi Xu for the helpful discussion on the related topics and the anonymous referee for his/her careful reading of the paper and for the comments and suggestions.

The work of H. Tian's team at PKU is supported by the National Natural Science Foundation of China(NSFC) under contracts 40574078 and 40436015. $\mathrm{H}$. Tian is now supported by China Scholarship Council for his stay in Germany. L.-D. Xia is supported by NSFC under Grant 40574064 and the Programme for New Century Excellent Talents in University (NCET).

\section{References}

Banerjee, D., O’Shea, E., \& Doyle, J. G. 2000, Sol. Phys., 196, 63

Brynildsen, N., Kjeldseth-Moe, K., Maltby, P., \& Wilhelm, K. 1999, ApJ, 517, L159
Cauzzi, G., Falchi, A., \& Falciani, R. 2000, A\&A, 357, 1093

Curdt, W., \& Heinzel, P. 1998, ApJ, 503, L95

Damé, L., Gouttebroze, P., \& Malherbe, J.-M. 1984, A\&A, 130, 331

DeForest, C. E., \& Gurman, J. B. 1998, ApJ, 501, L217

De Moortel, I., Ireland, J., \& Walsh, R. W. 2000, A\&A, 355, L23

De Moortel, I., Ireland, J., Hood, A. W., \& Walsh, R. W. 2002, A\&A, 387, L13

De Pontieu, B., Erdélyi, R., \& De Wijn, A. G. 2003, ApJ, 595, L63

De Pontieu, B., Erdélyi, R., \& James, S. P. 2004, Nature, 430, 536

De Pontieu, B., Erdélyi, R., \& De Moortel, I. D. 2005, ApJ, 624, L61

Dowdy, J. F. Jr., Rabin, D., \& Moore, R. L. 1986, Sol. Phys., 105, 35

Doyle, J. G., Popescu, M. D., \& Taroyan, Y. 2006, A\&A, 446, 327

Fludra, A. 1999, A\&A, 344, L75.

Fossum, A., \& Carlsson, M. 2005, Nature, 435, 16

Gabriel, A. H. 1976, Philos. Trans. R. Soc. London A, 281, 575

Hansteen, V. H., De Pontieu, B., Rouppe van der Voort, L., van Noort, M., \& Carlsson, M. 2006, ApJ, 647, L73

Jefferies, S. M., McIntosh, S. W., Armstrong, J. D., et al. 2006, ApJ, 648, L151

Kariyappa, R., \& Varghese, B. A. 2008, A\&A, 485, 289

Krijger, J. M., Rutten, R. J., Lites, B. W., et al. 2001, A\&A, 379, 1052

Lites, B. W., Rutten, R. J., \& Kalkofen, W. 1993, ApJ, 414, 345

McAteer, R. T. J., Gallagher, P. T.,Williams, D. R., et al. 2002, ApJ, 567, L165

McAteer, R. T. J., Gallagher, P. T., Bloomfield, D. S., et al. 2004, ApJ, 602, 436

McIntosh, S. W., \& Judge, P. G. 2001, ApJ, 561, 420

McIntosh, S. W., \& Jefferies, S. M. 2006, ApJ, 647, L77

Ofman, L., Nakariakov, V. M., \& DeForest, C. E. 1999, ApJ, 514, 441

O'Shea, E., Banerjee, D., \& Doyle, J. G. 2007, A\&A, 463, 713

Patsourakos, S., Vial, J.-C., Gabriel, A. H., \& Bellamine, N. 1999, ApJ, 522, 540

Peter, H. 2001, A\&A, 374,1108

Peter, H. 2004, Rev. Mod. Astron., 17, 87

Popescu, M. D., Banerjee, D., O’Shea, Doyle, J. G., \& Xia, L. D. 2005, A\&A, 442, 1087

Sakurai, T., Ichimoto, K., Raju, K.P., \& Singh, J. 2002, Sol. Phys., 209, 265

Srivastava, A. K., Kuridze, D., Zaqarashvili, T. V., \& Dwivedi, B. N. 2008, A\&A, 481, L95

Tian, H., Marsch, E., Tu, C.-Y., Xia, L.-D., \& He, J.-S. 2008a, A\&A, 482, 267

Tian, H., Xia, L.-D., \& Li, S. 2008b, A\&A, in press

Torrence, C., \& Compo, G.P., 1998. Bull. Amer. Meteor. Soc. 79, 61

Ugarte-Urra, I., Doyle, J. G., Madjarska, M. S., \& O'Shea, E. 2004a, A\&A, 418, 313

Ugarte-Urra, I., Doyle, J. G., Madjarska, M. S., \& Foley, C. R. 2004b, A\&A, 425,1083

Vecchio, A., Cauzzi, G., Reardon, K. P.,Janssen, K., \& Rimmele, T. 2007, A\&A, 461, L1

Withbroe, G. L., \& Noyes, R. W. 1977, ARA\&A, 15, 363

Xia, L.-D., Popescu, M. D., Doyle, J. G., \& Giannikakis, J. 2005, A\&A, 438, 1115 